\title{
Collective excitations in the continuum
}

\author{
G. G. Dussel \\ Departamento de Física, FCEyN-UBA, Ciudad Universitaria, Pabellón 1, 1428 Buenos Aires, Argentina \\ R. Id Betan \\ Departamento de Química y Física, FCEIA (UNR) - Instituto de Física Rosario, \\ IFIR (CONICET), Avenida Pellegrini 250, 2000 Rosario, Argentina \\ R. J. Liotta \\ KTH, Alba Nova University Center, SE-10691 Stockholm, Sweden \\ T. Vertse \\ Institute of Nuclear Research of the Hungarian Academy of Sciences, \\ H-4001 Debrecen, PO Box 51, Hungary and University of Debrecen, \\ Faculty of Informatics, H-4010 Debrecen, PO Box 12, Hungary
}

(Dated: November 25, 2009)

\begin{abstract}
Pairing (particle-particle) giant resonances are analyzed within a shell model formalism in the complex energy plane with the aim of understanding why they have not been observed so far. A comparison is made with the equivalent particle-hole mode by applying the formalism to the analysis of the well understood particle-hole giant resonance. It is found that due to the proper treatment of the continuum intrinsic to the formalism giant pairing resonances lie much higher than previously predicted and that some of them may be too wide to be observed while others are meaningful excitations. For these new experimental searches are proposed.
\end{abstract}

\section{INTRODUCTION}

Particle-hole (ph) and particle-particle (pp) modes are similar to each other, although their manifestations are apparently unrelated. In fact, ph excitations induce surface vibrations while pp induce pairing vibrations. In general the properties of the ph modes are manifested in the three-dimensional physical space while properties related to the pp modes are manifested in the so-called gauge space [1]. One may thus expect that the most collective of the ph vibrational modes, i. e. the giant resonance, would also be found in the pp channel as a giant pairing resonance which would be strongly excited by two-particle transfer reactions [2]. And indeed, using the same RPA formalism which describes well the ph giant resonances, it was found that pairing giant resonances would be found at about $10 \mathrm{MeV}$ of excitation energy in even-even nuclei $[3,4]$. In this standard formalism the representation used to write the RPA equations consists of bound single-particle states, for instance harmonic oscillator or the so-called Sturm-Louville states used in Ref. [4]. There is an important drawback with this kind of representations since it does not take into account the unstability of the single-particle states. In other words, processes occurring in the continuum are time dependent, although this dependence can be circumvented if the system lives a long time. This happens with the ph giant resonance, where bound representations explain very well properties related to the giant resonance, as will be discussed below. The pairing giant resonances, which were predicted to be strongly populated by two-particle transfer reactions in calculations performed within bound representations [4], have not been observed so far. Yet the pairing giant resonances are still object of much interest, both as excitations to be observed by using radioactive beams, which would avoid Q-value mismatchings at the high energies where the giant resonance would lie [5], and as a result of the clustering and associated strong coupling of Cooper pairs in the nuclear surface [6].

In this paper we will evaluate giant pairing resonances using as representation the eigenstates of a Wood-Saxon potential obtained as outgoing solutions of the Schrödinger equation. This is the Berggren representation [7]. It includes the bound states plus states in the complex energy plane that correspond to the Gamow resonances and scattering states. The Berggren representation is described in Section II. In Section III ph and pp giant resonances are evaluated. Possible experimental probes that may detect the giant pairing resonances are suggested in Section IV and a Summary and Conclusions are in the last Section.

\section{BERGGREN REPRESENTATION}

We will describe the giant resonances by using the Berggren representation. Although this is a subject which has been very much discussed in the literature recently $[8,9]$ we will give a brief description of the main points and exhibit 
the most important equations in order to facilitate the presentation.

The study of processes taking part in the continuum part of the spectrum may require, by the very nature of the problem, a time dependent formalism. However if there is a barrier that traps the system during a time long enough, the system will remain localized within the region of the barrier and the dynamics of the process can be studied within stationary formalisms. The physical meaning of terms like resonances living a "long time" or a barrier being "high enough" was discussed in detail in Ref. [10]. The main point is that one solves the time-independent Schrödinger equation imposing to the wave function outgoing boundary conditions. The energies thus obtained are real if the states are bound or antibound and complex if they are resonances. We will not deal here with antibound states.

Berggren found that these complex eigenvectors can be used to express the Dirac $\delta$-function as [11].

$$
\delta\left(r-r^{\prime}\right)=\sum_{n} w_{n}(r) w_{n}\left(r^{\prime}\right)+\int_{L^{+}} d E u(r, E) u\left(r^{\prime}, E\right)
$$

where the sum runs over all the bound states plus the complex states (resonances) which lie between the real energy axis and the integration contour $L^{+}$. The wave functions of the bound state or resonance $n$ is $w_{n}(r)$ and $u(r, E)$ is the scattering function at energy $E$. Notice that in this equation the scalar product between two functions is not a function times the complex conjugate of the other but just the product of the two. This is the Berggren metric and the corresponding vectors span the Berggren space. In the Berggren space energies and probabilities can become complex. We thus find that forcing the time-dependent process of particles interacting in the continuum to be stationary one has to pay the price of having complex energies and complex probabilities. Of all these complex states the ones which represent physically meaningful resonances are those which are localized and the corresponding complex probabilities are almost real. This corresponds to resonances that live a long time and the states can be considered quasi-bound. For a discussion on the limits of this approximation see Ref. [8].

Discretizing the integral of Eq. (1) one obtains the set of orthonormal vectors $\left|\varphi_{j}\right\rangle$ forming the Berggren representation [7]. Since this discretization provides an approximate value of the integral, the Berggren vectors fulfill the relation $I \approx \sum_{j}\left|\varphi_{j}\right\rangle\left\langle\varphi_{j}\right|$. These vectors include the set of bound states, Gamow resonances and discretized scattering states. With $\varphi_{j}(\vec{r})=\left\langle\varphi_{j} \mid \vec{r}\right\rangle$, in the applications below we will not show the radial wave function $\varphi_{j}(r)$ but rather the standard function $\phi_{j}(r)$ given by,

$$
\phi_{j}(r)=r \varphi_{j}(r)
$$

With the standard shell model Hamiltonian written as

$$
H=H_{0}+V
$$

where $H_{0}$ is the central field that we will choose as a Woods-Saxon potential and $V$ the residual interaction as described below (Eq. (7)), the single-particle states are given by

$$
H_{0}\left|\varphi_{j}>=\epsilon_{j}\right| \varphi_{j}>
$$

Using the Berggren representation one readily gets the two-particle shell-model equations in the complex energy plane (CXSM) [12], i. e.

$$
\left(\omega_{\alpha}-\epsilon_{i}-\epsilon_{j}\right) X(i j ; \alpha)=\sum_{k \leq l}<\tilde{k} \tilde{l} ; \alpha|V| i j ; \alpha>X(k l ; \alpha)
$$

where $V$ is the residual interaction, $\alpha$ labels two-particle states and $i, j, k, l$ label single-particle states. Therefore $\omega_{\alpha}$ is the correlated two-particle energy and $\epsilon_{i}$ is the single-particle energy corresponding to the state $i$. The two-particle wave function is given by

$$
\left|\alpha>=\sum_{i \leq j} X(i j ; \alpha) \frac{\left(c_{i}^{+} c_{j}^{+}\right)_{\lambda_{\alpha}}}{\sqrt{1+\delta_{i j}}}\right| 0>
$$

where $\lambda_{\alpha}$ is the angular momentum of the two-particle state.

The tilde in the interaction matrix element denotes mirror states so that in the corresponding radial integral there is not any complex conjugate, as required by the Berggren metric. Notice that this implies that the form of the CXSM equations coincides with the one corresponding to an harmonic oscillator representation, where all functions can be chosen to be real. 
We will use a separable interaction of the form

$$
\langle\tilde{k} \tilde{l} ; \alpha|V| i j ; \alpha\rangle=-G_{\alpha} f(k l, \alpha) f(i j, \alpha)
$$

where the constant $G_{\alpha}$ is the strength of the force, the function $f$ is $f(a b, \alpha)=$ $(-)^{l_{a}}\left\langle j_{a}\left\|Y_{\alpha}\right\| j_{b}\right\rangle \int \varphi_{a}(r) U(r) \varphi_{b}(r) r^{2} d r /\left(1+\delta_{a b}\right)^{1 / 2}$ and the potential $U$ is the derivative of the mean field used to determine the single-particle states which, in our case, will be a Woods-Saxon potential. The energies are obtained by solving the dispersion relation

$$
-\frac{1}{G_{\alpha}}=\sum_{i \leq j} \frac{f^{2}(i j, \alpha)}{\omega_{\alpha}-\left(\epsilon_{i}+\epsilon_{j}\right)}
$$

and the two-particle wave function amplitudes are given by

$$
X(i j ; \alpha)=N_{\alpha} \frac{f(i j, \alpha)}{\omega_{\alpha}-\left(\epsilon_{i}+\epsilon_{j}\right)}
$$

where $N_{\alpha}$ is the normalization constant determined by the condition $\sum_{i \leq j} X(i j ; \alpha)^{2}=1$.

One important quantity in the study to be performed below is the singlet $(\mathrm{S}=0)$ component of the two-particle wave function because for collective pairing states it shows clustering features [3]. With standard notation this function can be written as,

$$
\Psi_{\alpha J M}\left(\bar{r}_{1} \bar{r}_{2}\right)=\left[\chi_{1 / 2}(1) \chi_{1 / 2}(2)\right]_{0}^{0} \sum_{a \leq b} X(a b, \alpha J M) \hat{j}_{a} \hat{j}_{b}\left[C\left(a b, \bar{r}_{1} \bar{r}_{2}\right)-(-)^{j_{a}+j_{b}-J} C\left(b a, \bar{r}_{1} \bar{r}_{2}\right)\right]
$$

where,

$$
C\left(a b, \bar{r}_{1} \bar{r}_{2}\right)=\phi_{a}\left(r_{1}\right) \phi_{b}\left(r_{2}\right)(-)^{l_{b}+1 / 2-j_{a}+J}\left\{\begin{array}{ccc}
l_{a} & j_{a} & 1 / 2 \\
j_{b} & l_{b} & J
\end{array}\right\}\left[Y_{l_{a}}\left(\hat{r}_{1}\right) Y_{l_{b}}\left(\hat{r}_{2}\right)\right]_{J}^{M}
$$

and $\phi_{a}(r)$ is the radial wave function corresponding to the single-particle state $a$ (Eq. (2)). Notice that the dimension of the function $\Psi$ is $f m^{-2}$.

We would like to emphasize that we will evaluate all resonances that can be built within our Berggren single-particle representation and give physical meaning to the ones with wave functions showing localization properties and small imaginary parts within the nuclear volume.

\section{GIANT RESONANCES}

The shell model hereby presented will be used in the Tamm-Dancoff approximation (TDA) for pp states. For ph states, on the contrary, random-phase approximation (RPA) correlations are important, so the RPA formalism will be used to determine ph states. Therefore in this Section we will use the Berggren representation to study giant resonances within the TDA for the pp case and the RPA for the ph case. We will start with the ph excitations because here the bulk properties of the giant resonance are well explained by bound representations and therefore its analysis can help us to understand the influence of the continuum upon the resonance.

\section{A. The particle-hole giant resonance in the nucleus ${ }^{208} \mathbf{P b}$}

The first application of the Berggren representation, but excluding the scattering states on the complex contour, was performed just in the study of ph giant resonances in ${ }^{208} \mathrm{~Pb}$ [13]. Since only bound states and resonances were included in the representation the corresponding ph equations were called Resonant RPA (RRPA). It was thus found that the RRPA explains very well the bulk properties of the giant resonances, as bound representations had done before. The reason for this agreement between the RRPA and bound representations is that the main components of the giant resonance wave function have the spins of the particle and the hole aligned with the resonance angular momentum. This enhances the collectivity of the resonance because the angular momentum recoupling coefficients acquire in this case their maximum values. Moreover, the largest transition matrix elements are those for which the overlap between the particle and hole radial wave functions is largest. This occurs if the number of nodes in those wave functions is the same. Since the hole is in a low spin the alignment requires that the particle moves in a high 
spin orbit. Therefore the corresponding centrifugal barrier trap the system inside the nuclear volume hindering the decay of the resonance. In other words, the ph giant resonance is built upon single-particle resonances that are very narrow. These are quasi-bound single-particle states that are very well described by potentials that bind the particle (e. g. the harmonic oscillator potential).

This success of bound representations is limited to the explanation of the bulk properties of the giant resonance, like e. g. energies and sum rules. These are properties closely related to the matrix elements of the interaction or transition operators mentioned above. But when the proper continuum plays a role, like in the evaluation of the partial decay widths of the decaying resonance, then bound representations are not well-suited. This was shown in Ref. [14], where the continuum RPA equations were solved in the complex energy plane including also the continuum scattering waves. It was thus shown that for narrow resonances the total escape width (which is minus twice the imaginary part of the energy) is the sum of the partial escape widths. This condition was fulfilled by the giant resonances, indicating that a proper criterion to decide whether a resonance is meaningful is that the corresponding radial function is localized and that the corresponding imaginary part is small. We will use this criterion here not only to probe its validity by comparing with known excitations but also to learn how far a physical resonance is localized and how real is its wave function. Afterwards we will apply this criterion in the study of giant pairing resonances (GPR) to decide whether the evaluated states have physical relevance.

We will perform the calculations following the RRPA procedure of Ref. [13] where a separable force was used. However, our Berggren representation contains not only bound states and resonances but also scattering states in the complex energy plane and, therefore, we will call the formalism complex RPA (CXRPA). The results thus obtained should coincide with those given in Ref. [14] since, as mentioned above, in this reference the scattering states were also included, although within a continuum RPA calculation.

As it was the case of the CXSM the form of the CXRPA equations is the standard one. That is, the dispersion relation has the form

$$
\frac{1}{\kappa_{\alpha}}=\sum_{p h} \frac{2\left(\epsilon_{p}-\epsilon_{h}\right) f^{2}(p h, \alpha)}{\omega_{\alpha}^{2}-\left(\epsilon_{p}-\epsilon_{h}\right)^{2}}
$$

where $p(h)$ labels particle (hole) states, $\alpha$ labels the correlated ph state and $\kappa_{\alpha}$ is the strength of the multipolemultipole force. The function $f$ is the component of the separable force (cf. Eq. (7)) and the amplitude of the particle-hole wave function is $\left\langle\alpha\left|\left(c_{i}^{+} b_{j}^{+}\right)_{\lambda_{\alpha} \mu_{\alpha}}\right| 0\right\rangle=N_{\alpha}\left(n_{j}-n_{i}\right) f(i j, \alpha) /\left(\omega_{\alpha}-\left(\epsilon_{i}-\epsilon_{j}\right)\right)$, where $b_{j m}^{+}=(-1)^{j-m} c_{j-m}$ is the hole creation operator. Notice that the system is spherically symmetric and, therefore, the dependence upon $\mu_{\alpha}$ in these equations is formal only. One usually adopts the value $\mu_{\alpha}=0$. The occupation numbers is $n_{i}=1\left(n_{i}=0\right)$ if $i$ is a hole (particle) state. The particle-hole energy $\epsilon_{p h}=\epsilon_{p}-\epsilon_{h}$ is a positive quantity for bound states, where all energies are real. In our case the energies can be complex, but still the real part of $\epsilon_{p h}$ is positive. With this notation one can write the QXRPA wave function in the standard form, i. e. $\mid \alpha>=\sum_{p h}\left(X(p h ; \alpha)\left(c_{p}^{+} b_{h}^{+}\right)_{\lambda_{\alpha} \mu_{\alpha}} \mid 0>\right.$ $\left.-(-1)^{\lambda_{\alpha}} Y(p h ; \alpha)\left(c_{h}^{+} b_{p}^{+}\right)_{\lambda_{\alpha} \mu_{\alpha}} \mid 0>\right)$, where $X(p h ; \alpha)=<\alpha\left|\left(c_{p}^{+} b_{h}^{+}\right)_{\lambda_{\alpha} \mu_{\alpha}}\right| 0>=N_{\alpha} f(p h, \alpha) /\left(\omega_{\alpha}-\epsilon_{p h}\right)$ (forward amplitude) and $Y(p h ; \alpha)=<\alpha\left|\left(c_{h}^{+} b_{p}^{+}\right)_{\lambda_{\alpha} \mu_{\alpha}}\right| 0>=-N_{\alpha} f(h p, \alpha) /\left(\omega_{\alpha}+\epsilon_{p h}\right)$ (backward amplitude). The normalization constant $N_{\alpha}$ is obtained through the RPA condition $\sum_{p h}\left(X^{2}(p h, \alpha)-Y^{2}(p h, \alpha)\right)=1$, as usual.

Also in the particle-hole analysis to be performed below the $\mathrm{S}=0$ component of the wave functions will be examined to probe the clustering features of the states. This component can be evaluated as for the two-particle case and the corresponding expression is similar to the one obtained there (Eq. (10)).

We will evaluate the ph resonances by repeating the RPA calculation of Ref. [14], where the giant resonances corresponding to angular momenta $\lambda \leq 3$ were analyzed. The Hamiltonian will be as described above, i. e. the mean field will be a Woods-Saxon potential and the residual interaction will be the separable force Eq. (7). Of all these resonances we will only show the dipole one because the conclusions from this case are also valid for the quadrupole $(\lambda=2)$ and octupole $(\lambda=3)$ cases. Our main purpose in this study is to explore the clustering properties of the giant resonance. With this in mind we notice that the wave function depends upon six independent coordinates. Thus, in spherical coordinates the wave function is $\Psi\left(\vec{r}_{p}, \vec{r}_{h}\right)$ where the coordinate of the particle is $\vec{r}_{p}=\left(r_{p}, \theta_{p}, \varphi_{p}\right)$ and the same for the hole radius $\vec{r}_{h}$. Due to spherical symmetry (since the mean field as well as the residual interaction are spherically symmetric) the dependence upon $\varphi$ is irrelevant. That is, the wave function itself depends upon the angles $\varphi_{p}$ and $\varphi_{h}$ but the physical quantities (e. g. probabilities) do not. Moreover, in order to examine the clustering features on the nuclear surface it is enough to show the singlet component of $\Psi\left(\vec{r}_{p}, \vec{r}_{h}\right)$ (Eq. (10)) as a function of the angle between $\vec{r}_{p}$ and $\vec{r}_{h}$. We therefore take $r_{p}=r_{h}=R$, where $R$ is the nuclear radius, which in ${ }^{208} \mathrm{~Pb}$ we choose as $R=7.2 \mathrm{fm}$. In addition we take $\vec{r}_{p}$ to coincide with the $z$-axis, i. e. $\theta_{p}=0$. With this choice of coordinates the wave function $\Psi(\theta)$ depends only upon the angle $\theta$ between $\vec{r}_{p}$ and $\vec{r}_{h}$, which is $\theta=\theta_{h}$. As we will see $\Psi\left(\vec{r}_{p}, \vec{r}_{h}\right)$ is clustered, i. e. it is peaked at $\theta=0$. Therefore to show the localization as well as the extend to which the wave 


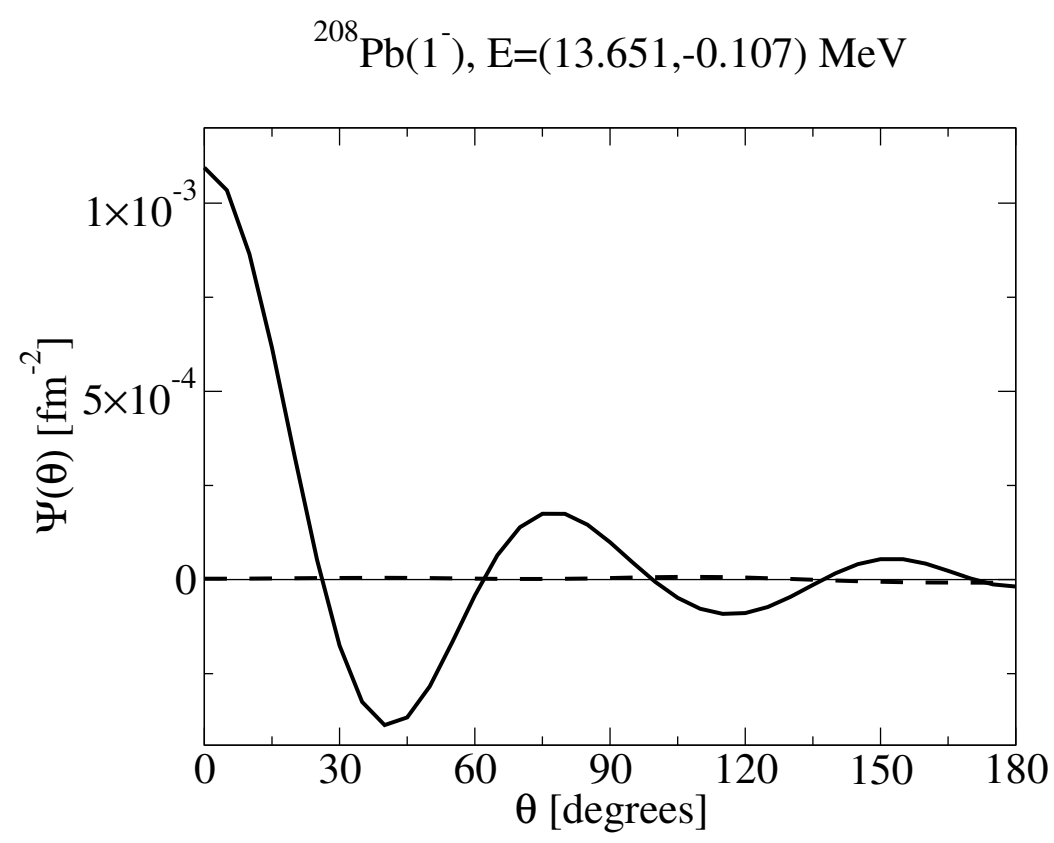

FIG. 1: Particle-hole wave function $\Psi(\theta)$ corresponding to the giant dipole resonance in ${ }^{208} \mathrm{~Pb}$. $\theta$ is the angle between the particle and hole radii. The particle radii $\vec{r}_{p}$ coincides with the $z$-axis. Both radii are taken at $r_{p}=r_{h}=7.2 \mathrm{fm}$. Full (dashed) line represents the real (imaginary) part of the wave function.

function is real we choose $\theta=0, r_{p}=r_{h}=r$ and plot $\Psi(r)$ as a function of $r$. This is the choice used in Ref. [8] to determine those features, i. e. whether a given resonance was meaningful.

In Fig. 1 we show the wave function $\Psi(\theta)$ corresponding to the giant dipole resonance in ${ }^{208} \mathrm{~Pb}$ which lie at the complex energy $E=(13.651,-0.107) \mathrm{MeV}$. One sees, as expected, that the giant dipole resonance is indeed peaked at $\theta=0$ and that it is practically a real function. This will serve us as an example of a state lying deep into the continuum and which is a physically meaningful resonance.

In Fig. 2 we show $\Psi(r)$. Here the imaginary part of the wave function is also negligible, except very much inside the nucleus where it acquires a small value. But one sees that the wave function is indeed localized inside the nuclear volume, as required for a complex state to be meaningful. Also this case will be taken as an example in the next Section to decide whether it would be worthwhile to search experimentally for the calculated giant pairing resonances.

Another important feature is to note that the escape width of the dipole giant resonance is $214 \mathrm{keV}$. This is only a fraction of the total width $\Gamma$ of the observed resonance, which is about $2 \mathrm{MeV}$. Most of the width $\Gamma$ is provided by the spreading width, i. e. by more complicated configurations than the ph ones included in the RPA calculation performed here. These complicated configurations are mostly of a two-particle two-hole character [15, 16].

\section{B. The particle-particle pairing giant resonances outside the nucleus ${ }^{208} \mathbf{P b}$}

We are now in a position to evaluate the $\mathrm{T}=1$ two-particle giant pairing resonances outside the ${ }^{208} \mathrm{~Pb}$ core. As pointed out above, the RPA correlations are negligible in these two-particle excitations and therefore one generally uses the TDA formalism to study them, as we do below.

These are excitations lying high in the nuclear spectrum which would be highly excited by two-particle transfer probes. To analyze these excitations we will also use the coordinates chosen in the ph case above. With $\vec{r}_{i}, i=1,2$, the particle $i$ has the position $\vec{r}_{i}=\left(r_{i}, \theta_{i}, \varphi_{i}\right)$. The clustering of the pairing giant resonance wave function $\Psi\left(\vec{r}_{1}, \vec{r}_{2}\right)$ will be analyzed by locating the particle $\vec{r}_{1}$ along the $z$-axis and defining $\theta=\theta_{2}$ with $r_{1}=r_{2}=R=7.2 \mathrm{fm}$. The localization and the complex character of $\Psi\left(\vec{r}_{1}, \vec{r}_{2}\right)$ will be probed by using, as above, $r_{1}=r_{2}=r$ and $\theta=0$, thus procuring that $\Psi\left(\vec{r}_{1}, \vec{r}_{2}\right)$ depends only upon $r$. 


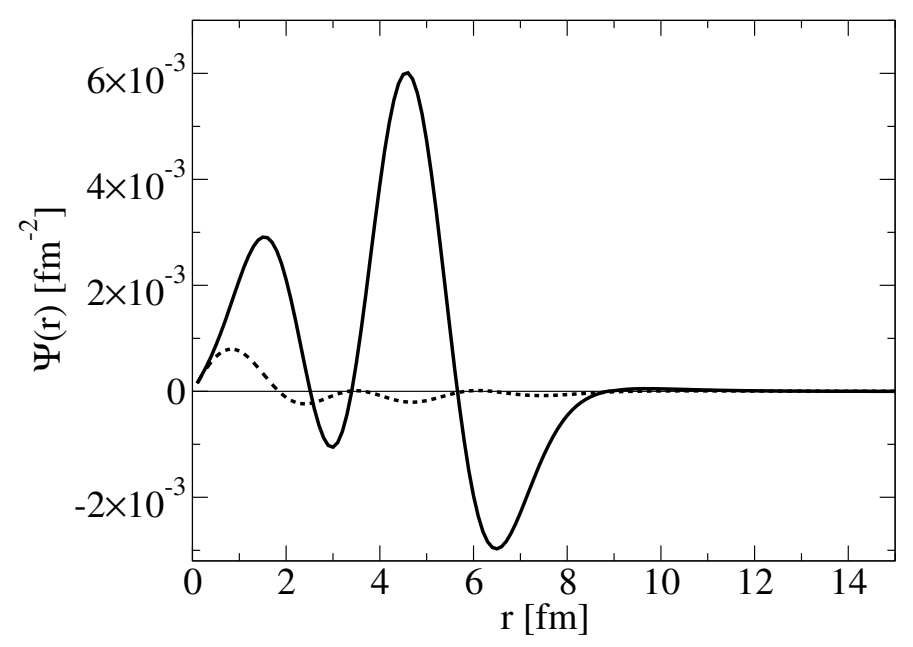

FIG. 2: Particle-hole wave function $\Psi(r)$ corresponding to the giant dipole resonance in ${ }^{208} \mathrm{~Pb}$. The distance $r=r_{p}=r_{h}$ is taken at the angle $\theta=0$ (see text). Full (dashed) line represents the real (imaginary) part of the wave function.

As shown in Ref. [4] the two-particle form factor entering the corresponding two-particle transfer cross section is proportional to the two-particle wave function $\Psi(\theta=0)$ at the nuclear surface, i. e. the wave function that we use to show clustering at its peak. That is the reason why the giant pairing resonance is strongly excited in two-particle transfer reactions. That is, this resonance shows the highest clusterization of all two-particle states and also, as will be seen below, the highest peak.

We will only analyze monopole pairing vibrations, which are the most collective of all pairing states. In ${ }^{208} \mathrm{~Pb}$, with $\mathrm{Z}=82, \mathrm{~N}=126$, the $\mathrm{T}=1$ monopole isobaric analogous state to ${ }^{210} \mathrm{~Pb}(\mathrm{gs})$ are the states ${ }^{210} \mathrm{Bi}\left(0^{+} ; G P R\right)$ and ${ }^{210} \mathrm{Po}\left(0^{+} ; G P R\right)$.

We will analyze these three pairing states separately according to the value of $T_{z}$. The Woods-Saxon mean field will be as in the ph case above. The corresponding single-particle states, which we will mention often, are given in Table II of Ref. [13]. The two-body force will be the standard monopole pairing interaction of Ref. [4].

We will evaluate the state ${ }^{210} \mathrm{Po}\left(0^{+} ; G P R\right)$ by adjusting the pairing strength $\mathrm{G}$ of the separable force to obtain the experimental ${ }^{210} \mathrm{Po}(\mathrm{gs})$ energy. In ${ }^{210} \mathrm{Bi}$ there is not any proton-neutron $0^{+}$state which is experimentally known. We will therefore assume various values for $\mathrm{G}$ and investigate the clustering and localization features of the states trying to find the giant pairing resonance. We will thus sees that this resonance may actually be a bound state and that its emergence will depend strongly on its vicinity to the continuum threshold.

\section{C. $T_{z}=-1$ : the nucleus ${ }^{210} \mathbf{P o}$}

The ground state of ${ }^{210} \mathrm{Po}$ is the proton-proton vibrational state. This state was exhaustively analyzed in Ref. [4]. However in this reference a bound single-particle basis was used and, therefore, the states thus calculated do not coincide with those evaluated in our Berggren basis. Yet, ${ }^{210} \mathrm{Po}(\mathrm{gs})$ is a bound state and therefore the differences between our calculation and that of Ref. [4] for this state are minor. This is satisfactory due to the checking of our computer codes implied by the good agreement between the two calculations. Therefore this calculation is important, but our aim is to assess whether the giant pairing resonance indeed exists and in this case whether it can be observed. To achieve this we fitted the energy of ${ }^{210} \mathrm{Po}(\mathrm{gs})$ to obtain the value of the strength $\mathrm{G}$. With this value of $\mathrm{G}$ we then obtained the whole two-particle spectrum. Among the states thus found we obtained one which is strongly clustered at an energy of $\omega=(14.907,-0.009) \mathrm{MeV}$ ( $\omega$ is energy measured from ${ }^{210} \mathrm{Po}(\mathrm{gs})$ ). This very narrow state is shown in Fig. 3. The collectivity of this GPR can be assessed by comparing with the corresponding clustering in ${ }^{210} \mathrm{Po}(\mathrm{gs})$, which is shown in Fig. 4. One sees that indeed at $\theta=0$ the wave function of the high lying state is about 1.8 times the one corresponding to the ground state, and in the cross section it is the square of this number that enters. But the 


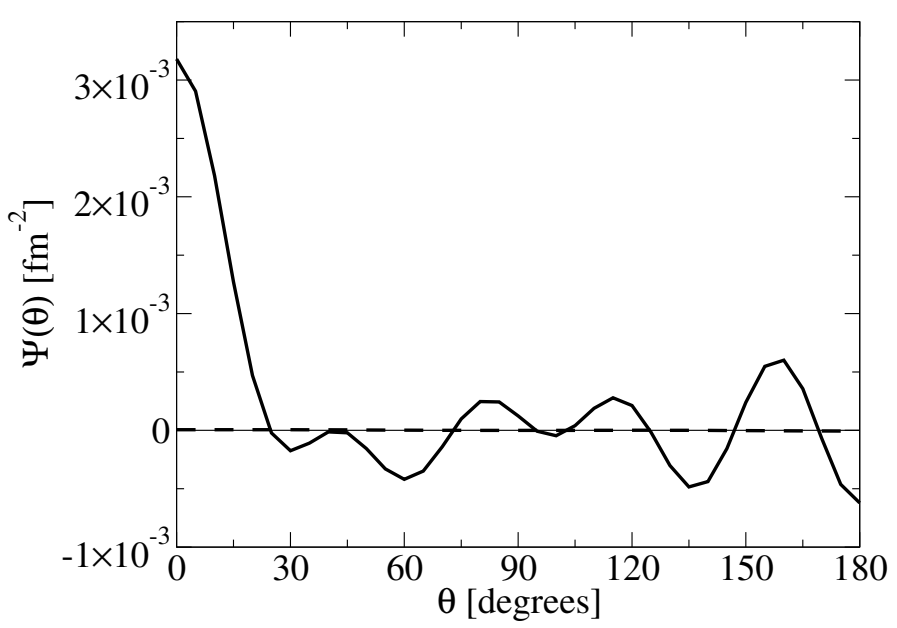

FIG. 3: ${ }^{210}$ Po GPR wave function. The angle $\theta$ between the two-particles and the positions of the particles are as indicated in the text. Full (dashed) line represents the real (imaginary) part of the wave function.

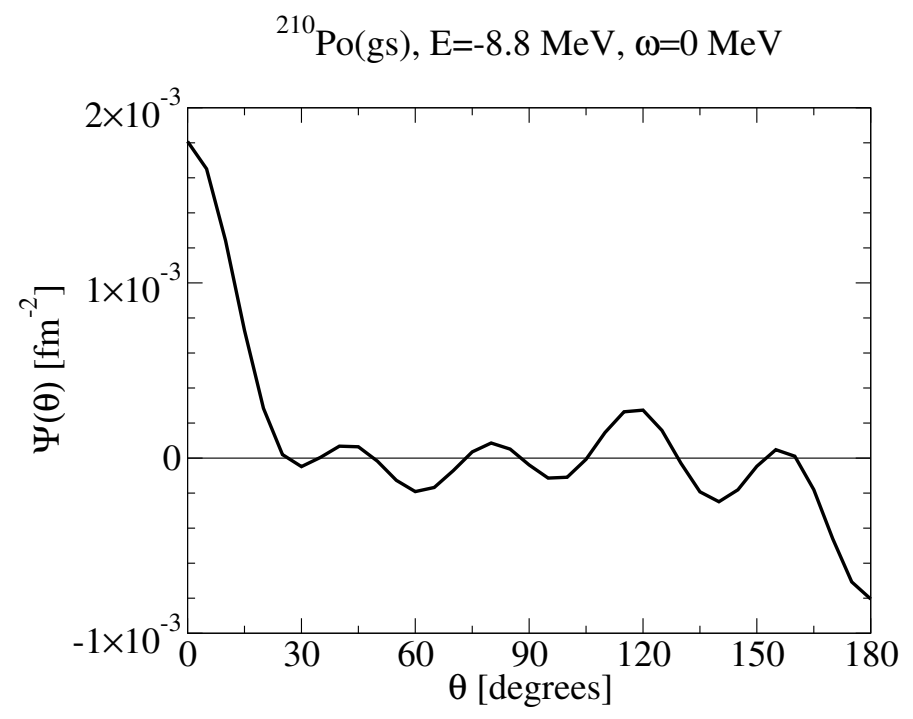

FIG. 4: As in Fig. 3 but for the ground state of ${ }^{210}$ Po.

striking feature in Fig. 3 is that the wave function is practically real. To analyze this point farther and, at the same time, to verify whether the state is localized, we show in Fig. 5 the radial dependence of the GPR wave function. We thus sees that the GPR in this nucleus is not only practically real but also that is as localized as any bound state would be. One can illustrate this point even more by comparing with the ph case of the previous Section. One thus sees that our giant pairing resonance is at least as physical as the dipole giant resonance in ${ }^{208} \mathrm{~Pb}$ shown in Fig. 2. The escape width is $18 \mathrm{keV}$ which, again comparing with the ph giant resonance, indicates that the total width may be only some hundreds of keV.

The reason why this GPR is so narrow is partly due to the Coulomb barrier, but also because it is largely built upon 


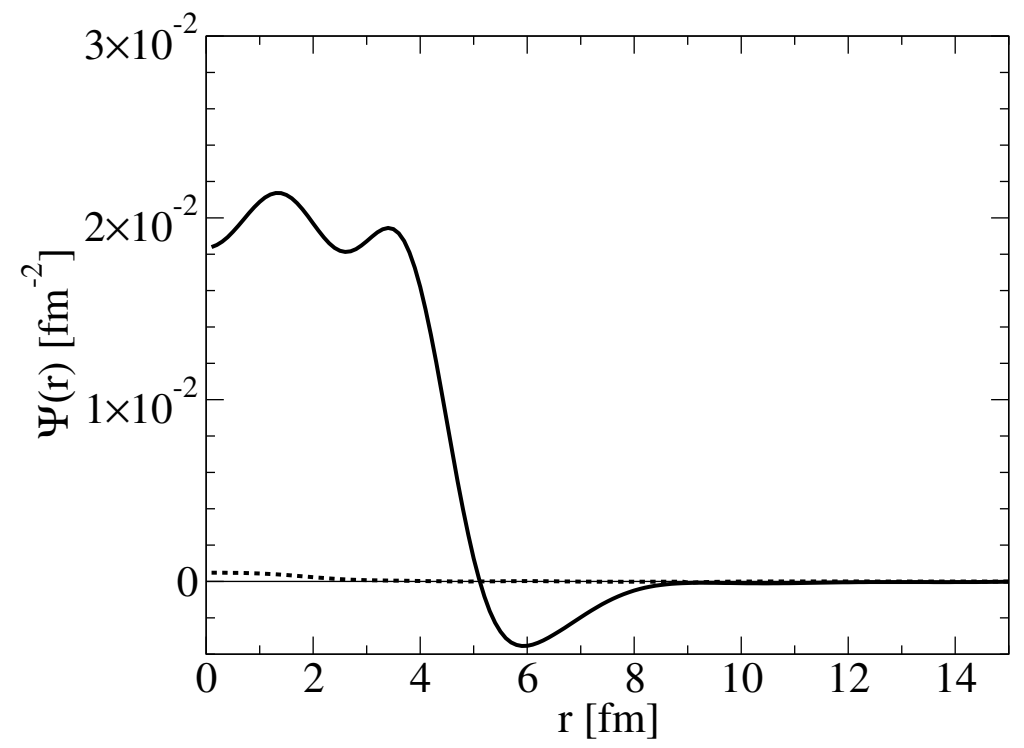

FIG. 5: ${ }^{210}$ Po GPR wave function. The distance $r$ is as indicated in the text. Full (dashed) line represents the real (imaginary) part of the wave function.

high spin configurations, i. e. upon very narrow single-particle states. Thus, the three most important of these states are $1 g_{9 / 2}$ lying at an energy of $(4.03,-0.00) \mathrm{MeV}, 0 j_{15 / 2}$ at $(5.96,-0.00) \mathrm{MeV}$ and $0 i_{11 / 2}$ at $(5.43,-0.00) \mathrm{MeV}$ (notice that this are energies measured from $\left.{ }^{208} \mathrm{~Pb}(\mathrm{gs})\right)$. These are high lying states, which explains why the GPR also lies very high in the spectrum, actually more than $3 \mathrm{MeV}$ higher than the $11.6 \mathrm{MeV}$ predicted in Ref. [4]. In spite of this, it seems that it is worthwhile to urge experimentalist groups to search in ${ }^{210} \mathrm{Po}$ for one of the most elusive collective states in nuclei. One suitable process to perform this search is two-particle transfer reactions. As an example of this type of reactions we show in Fig. 6 the angular distribution corresponding to the reaction ${ }^{208} \mathrm{~Pb}\left({ }^{3} \mathrm{He}, n\right){ }^{210} \mathrm{Po}(G P R)$ at a projectile energy of $100 \mathrm{MeV}$. This Figure can also be useful as a guide, since in two-particle transfer reactions with light projectiles the differential cross sections corresponding to different states with a given angular momentum are approximately proportional to each other. For the $0^{+}$states studied here the angular distribution is as seen in Fig. 6. That is, it has a typical very pronounced oscillating behaviour. For states with other angular momenta the pattern of the angular distribution is smoother. This can be seen in Fig. 3 of Ref. [18], where other details of two-particle transfer reaction cross sections are also discussed. The important point for us is that the angular distribution for the reaction leading to ${ }^{210} \mathrm{Po}(\mathrm{gs})$ is the same as the one in Fig. 6, but smaller but a factor of $1.8^{2} \approx 3$, as mentioned above.

\section{D. $T_{z}=0$ : the nucleus ${ }^{210} \mathrm{Bi}$}

In this nucleus the low-lying states correspond to configurations in which protons move in the $\mathrm{N}=5$ shell and neutrons in the $\mathrm{N}=6$ shell. Therefore most of the low-lying proton-neutron (pn) states are of negative parity. The first $0^{+}$pn state would consist of $\mathrm{N}=6$ configuration for both neutrons and protons, i. e. it would be the GPR where the low-lying neutron states are bound and the protons move in states lying in the continuum part of the spectrum.

There is not any pn $0^{+}$experimental state to be used in the determination of the strength $\mathrm{G}$. In fact if this state existed it would be the GPR, which we will assume to lie at a reasonable energy in order to investigate whether it may have any physical significance. We will therefore assume that the gap induced by the pairing interaction is about -1.5 $\mathrm{MeV}$, as it is in ${ }^{210} \mathrm{~Pb}(\mathrm{gs})$. Since the lowest GPR configuration is $\left(\pi 1 g_{9 / 2} \nu 1 g_{9 / 2}\right)_{0+}$ and $\epsilon\left(\pi 1 g_{9 / 2}\right)=(4.03,0.00) \mathrm{MeV}$, $\epsilon\left(\nu 1 g_{9 / 2}\right)=-3.93 \mathrm{MeV}$, the GPR should lie between $(0.03,0.00) \mathrm{MeV}$ (corresponding to vanishing pairing interaction) 


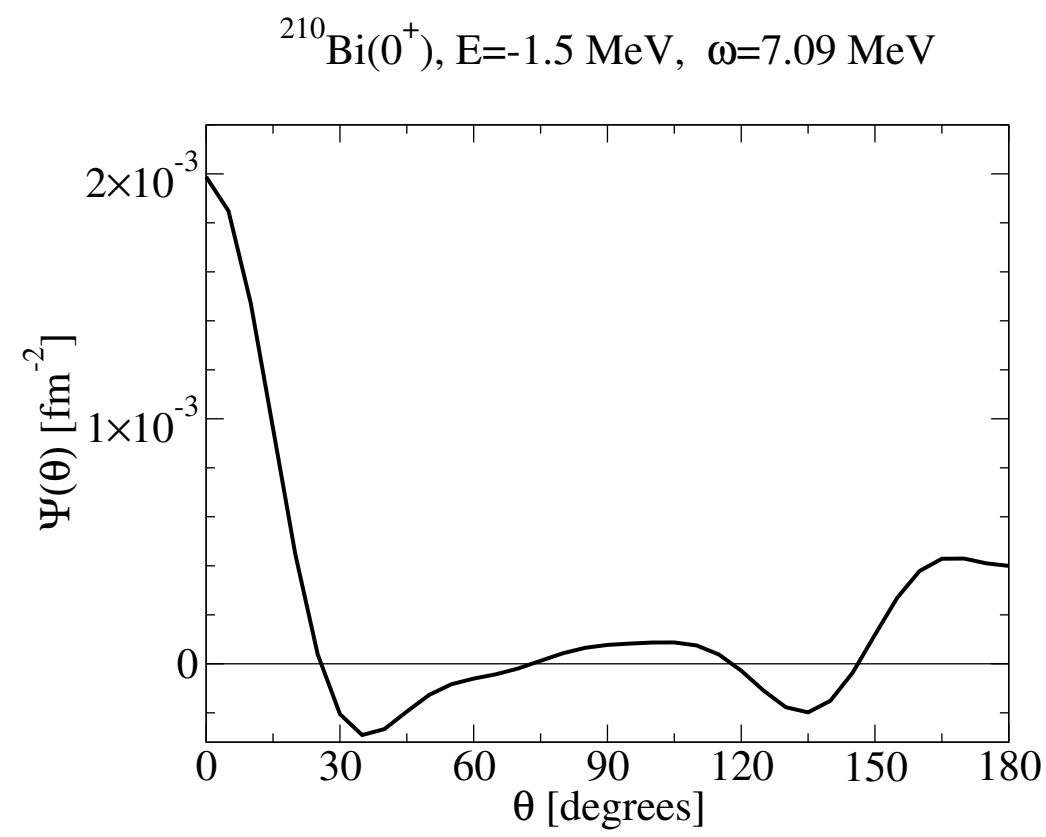

FIG. 7: ${ }^{210} \mathrm{Bi}$ GPR wave function corresponding to the case $E=-1.5 \mathrm{MeV}$. The angle $\theta$ is as indicated in the text. Notice that this is a bound state and therefore the wave function is real.

\section{PROBES TO DETECT THE GIANT PAIRING RESONANCES}

In this Section we will explore experimental possibilities to detect the GPR that we have studied above. Given the pairing collective character of these states the most obvious of the probes to be used would be two-particle transfer reactions. The theoretical analysis of the two-particle transfer reactions leading to these states has been performed in Ref. [4]. Of all the GPR studied in Ref. [4] (and in this paper as well) the only one which has been investigated experimentally, through the reaction ${ }^{208} \mathrm{~Pb}(\mathrm{t}, \mathrm{p})^{210} \mathrm{~Pb}$, was the one which we found to be too wide to be observed, i. e. ${ }^{210} \mathrm{~Pb}(\mathrm{GPR})$. That experiment [19] did not show any trace of the GPR. This is to be expected since the GPR is here too wide to be physically meaningful. This feature was not realized in Ref. [4] because in the corresponding calculations the continuum was ignored. However, we expect that the GPR in ${ }^{210} \mathrm{Bi}$ and specially in ${ }^{210} \mathrm{Po}$ may be found. We would therefore suggest that the corresponding neutron-proton (e. g. $(\alpha, \mathrm{d})$ ) and proton-proton (e.g. $\left.\left({ }^{8} \mathrm{Be},{ }^{6} \mathrm{He}\right)\right)$ reactions, which as far as we know have not been performed so far, may be proper tools to reach the giant pairing resonances.

Another independent way of probing the GPR is by exploiting the $\mathrm{T}=1$ isobaric character of the states ${ }^{210} \mathrm{Po}(\mathrm{GPR})$, ${ }^{210} \mathrm{Bi}(\mathrm{GPR})$ and ${ }^{210} \mathrm{~Pb}(\mathrm{gs})$. One thus expects that e. g. the reaction ${ }^{210} \mathrm{~Pb}(\mathrm{gs})(\mathrm{p}, \mathrm{n})$ would populate the state ${ }^{210} \mathrm{Bi}(\mathrm{GPR})$. This type of reactions, as well as $\left({ }^{3} \mathrm{He}, \mathrm{t}\right)$, is been used extensively to probe Gamow-Teller strengths, e. g. in Ref. [20]. However we have not found any publication where such reactions are performed by using ${ }^{210} \mathrm{~Pb}(\mathrm{gs})$ as a target. Again in this case we would encourage experimental groups to analyze this possibility, which would provide invaluable information about pairing collectivity in the continuum as well as on the continuum itself.

The corresponding reaction leading to the double analog state ${ }^{210} \mathrm{Po}(\mathrm{GPR})$, like e. g. $\left({ }^{8} \mathrm{Be},{ }^{8} \mathrm{He}\right)$, has not been performed either. But this interesting state was investigated long ago [21]. In this experiment the double analog state was detected as an enhancement in the excitation function for the reaction $p+{ }^{209} \mathrm{Bi} \rightarrow{ }^{208} \mathrm{~Pb}+2 p$. However, this experiment was not follow up by other investigations and the state thus observed is rarely mentioned nowadays. We do hope that the results presented in this paper would encourage experimental groups to gauge the possibility of performing a proper $(2 \mathrm{p}, 2 \mathrm{n})$ reaction experiment. 


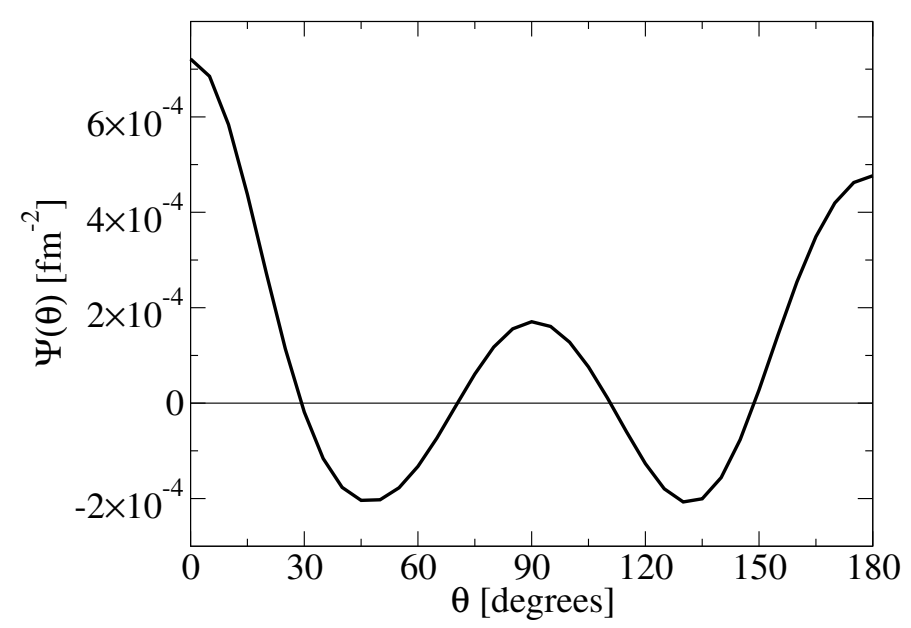

FIG. 8: ${ }^{210} \mathrm{Bi}$ GPR wave function corresponding to the case $E=-0.05 \mathrm{MeV}$. The angle $\theta$ is as indicated in the text.

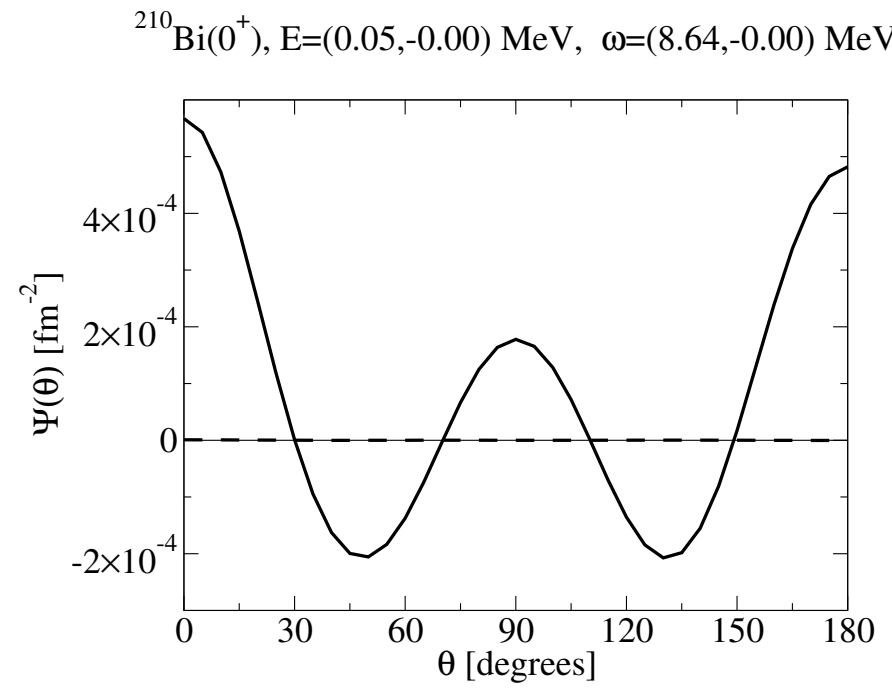

FIG. 9: ${ }^{210} \mathrm{Bi}$ GPR wave function corresponding to the case $E=(0.05,-0.00) \mathrm{MeV}$. The angle $\theta$ is as indicated in the text. Full (dashed) line represents the real (imaginary) part of the wave function.

\section{SUMMARY AND CONCLUSIONS}

In this paper we have analyzed monopole $\mathrm{T}=1$ giant pairing resonances (GPR) outside the ${ }^{208} \mathrm{~Pb}$ core. Our motivation for this study was to explore why these resonances, which have been studied thoroughly in the past (e. g. in Refs. $[4,5])$ and predicted to be highly excited by two-particle transfer probes, have not been observed so far. We noticed that those studies were performed within bound representations, which do not take into account the unstable character of continuum excitations. That is, those calculation did not consider the decaying nature of the resonances. We repeated the same calculations but using the Berggren representation, which allows one to evaluate shell model excitations in the complex energy plane. The imaginary parts of the energies thus evaluated are minus twice the 


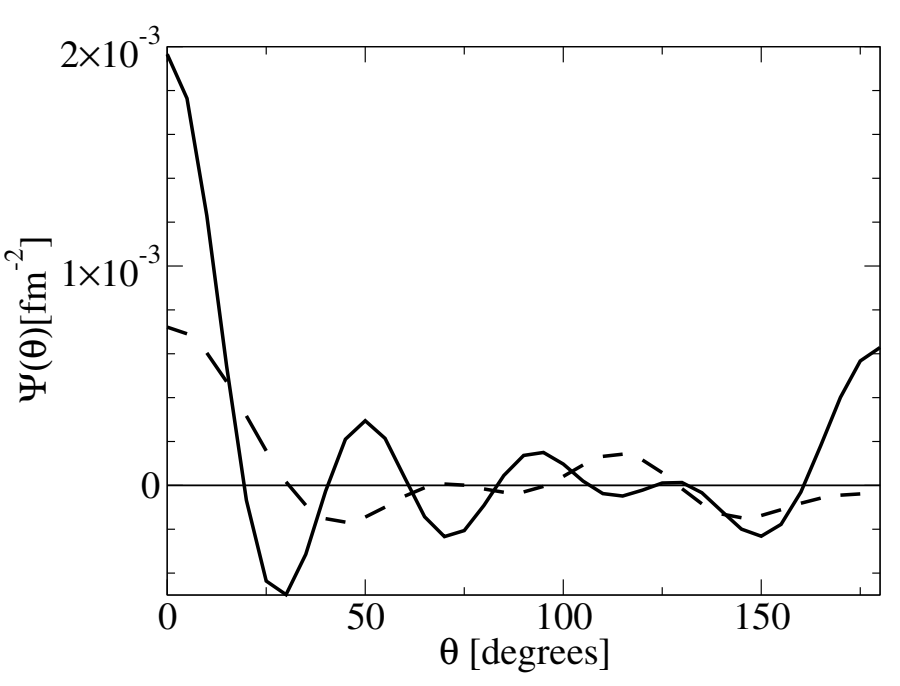

FIG. 10: ${ }^{210} \mathrm{~Pb}$ GPR wave function. The angle $\theta$ is as indicated in the text. Full (dashed) line represents the real (imaginary) part of the wave function.

escape widths of the resonances. We found that the two-neutron GPR in ${ }^{210} \mathrm{~Pb}$ is very wide and is not a physically relevant state, but rather a part of the continuum background. The proton-neutron GPR in ${ }^{210} \mathrm{Bi}$ is found to be a meaningful state only if it is not a resonance but a bound state lying below $7 \mathrm{MeV}$ of excitation. As this energy approaches the continuum threshold then the collectivity of the state gradually disappears. Above threshold not only the collectivity vanishes altogether but also the resulting resonance is very wide, as the one in ${ }^{210} \mathrm{~Pb}$ had shown to be. Finally, the proton-proton GPR in ${ }^{210}$ Po, lying at (14.907,-0.009) MeV, higher in the spectrum than previously predicted, turns out to be a meaningful state. The reason for this is that it is mainly built upon high lying and high spin, and therefore very narrow, single-particle proton states. It is also very collective and, therefore, it seems proper to urge experimental groups to search for this most elusive collective mode in ${ }^{210}$ Po. In this sense we have also proposed a number of experiments, ranging from two-particle transfer reactions to ( $\mathrm{p}, \mathrm{n})$ and $(2 \mathrm{p}, 2 \mathrm{n})$ experiments, which would allow to observe states analog to ${ }^{210} \mathrm{~Pb}(\mathrm{gs})$. These experiments just deal with the resonances that we found to be physically meaningful, i. e. ${ }^{210} \mathrm{Bi}(\mathrm{GPR})$ and specially ${ }^{210} \mathrm{Po}(\mathrm{GPR})$.

\section{Acknowledgments}

We want to express our gratitude to D. R. Bes and O. Civitarese for discussions. This work was supported in part by PIP-5287 (CONICET-Argentina).

[1] A. Bohr and B. Mottelson, Nuclear Structure, Vol.II, World Scientific Publishing Co., 1998, Singapore.

[2] R. Broglia and D. R. Bes, Phys. Lett. 68B, 129 (1977).

[3] M. W. Herzog, R. J. Liotta and L. J. Sibanda, Phys. Rev. C 31, 259 (1985).

[4] M. W. Herzog, O. Civitarese, L. S. Ferreira, R. J. Liotta, T. Vertse and L. J. Sibanda, Nucl. Phys. A 448, 441 (1986).

[5] L. Fortunato, W. von Oertzen, H. M. Sofia and A. Vitturi, Eur. Phys. J. A 14, 37 (2002).

[6] N. Pillet, N. Sandulescu and P. Schuck, Phys. Rev. C 76, 024310 (2007).

[7] R. J. Liotta, E. Maglione, N. Sandulescu and T. Vertse, Phys. Lett. 367B, 1 (1996).

[8] G. G. Dussel, R. Id Betan, R. J. Liotta and T. Vertse, Nucl. Phys. A 789, 182 (2007).

[9] N. Michel, W. Nazarewicz, M. Płoszajczak and T. Vertse, J. Phys. G 36, 013101 (2009).

[10] R. M. Id Betan, R. J. Liotta, N. Sandulescu, T. Vertse and R. Wyss, Phys. Rev. C 72, 054322 (2005).

[11] T. Berggren, Nucl. Phys. A 109, 265 (1968). 
[12] R. Id Betan, R. J. Liotta, N. Sandulescu and T. Vertse, Phys. Rev. C 67, 014322 (2003).

[13] P. Curutchet, T. Vertse and R. J. Liotta, Phys. Rev. C 39, 1020 (1989).

[14] T. Vertse, R.J. Liotta, and E. Maglione, Nucl. Phys. A584, 13 (1995).

[15] A. van der Woude, Prog. Part. Nucl. Phys. 18, 17 (1987).

[16] S. Gales, Ch. Stoyanov and A, I, Vdovin, Phys. Rep. 166, 127 (1988).

[17] R.E. Anderson, P.A. Batay-Csorba, R.A. Emigh, E.R. Flynn, D.A. Lind, P.A. Smith, C.D. Zafiratos and R.M. De Vries, Phys. Rev. C 19, 2138 (1979).

[18] W.A. Lanford, Phys. Rev. Cl6 (1977) 988.

[19] L. Zybert, Nuclear Structure, Daresburry Annual Report 1987-1988.

[20] R. G. T. Zegers et al, Phys. Rev. C 74, 024309 (2006).

[21] G. W. Hoffmann, G. J. Igo, C. A. Whitten, W. H. Dunlop and J. G. Kulleck, Phys. Rev. Lett. 28, 41 (1972). 